\title{
Adaptation of public health initiatives: expert views on current guidance and opportunities to advance their application and benefit
}

\section{Serene Yoong}

Hunter New England Local Health District

Katarzyna Bolsewicz ( $\sim$ katarzyna.bolsewicz@health.nsw.gov.au )

Hunter New England Local Health District

Alice Grady

Hunter New England Local Health District

Rebecca Wyse

Hunter New England Local Health District

\section{Rachel Sutherland}

Hunter New England Local Health District

Rebecca Kate Hodder

Hunter New England Local Health District

Melanie Kingsland

Hunter New England Local Health District

Nicole Nathan

Hunter New England Local Health District

\section{Sam McCrabb}

University of Newcastle

Adrian Bauman

University of Sydney

John Wiggers

Hunter New England Local Health District

Joanna Moullin

Curtin University

\section{Bianca Albers}

European Implementation Collaborative

\section{Maria Fernandez}

University of Texas Health Science Centre

Alix Hall

Hunter Medical Research Institute

Joanie Sims-Gould 
University of British Columbia

\section{Natalie Taylor}

Cancer Council New South Wales

\section{Chris Rissel}

NSW Office of Preventive Health

Andrew Milat

NSW Ministry of Health

Andrew Bailey

Mid North Coast Local Health District

\section{Samantha Batchelor}

Central Coast Local Health District

\section{John Attia}

University of Newcastle

\section{Luke Wolfenden}

Hunter New England Local Health District

\section{Research article}

Keywords: adaptation, evidence-based interventions, frameworks, qualitative

Posted Date: August 13th, 2019

DOI: https://doi.org/10.21203/rs.2.12693/v1

License: (c) (i) This work is licensed under a Creative Commons Attribution 4.0 International License. Read Full License

Version of Record: A version of this preprint was published at Health Education Research on July 6th, 2020. See the published version at https://doi.org/10.1093/her/cyaa014. 


\section{Abstract}

Background While there is some guidance to support the adaptation of evidence-based public health interventions, little is known about adaptation in practice and how to best support public health practitioners in its operationalization. This study explored public health researchers' and practitioners' views on available adaptation frameworks, identified potential improvements to increase the application of such guidance and identified opportunities to advance the adaptation literature to improve implementation of public health initiatives and achieve public health outcomes. Methods This qualitative study was undertaken with researchers, methodologists, policy makers and practitioners representing various public health expert organizations and universities internationally. Participants attended a face to face workshop in Newcastle, Australia in October 2018 where structured facilitated focus group discussions and small group activities were undertaken. Two qualitative methods - World Café and Appreciative Inquiry - were used to explore participants' experiences of and opinions about adaptation frameworks, and to elicit suggestions for practical, detailed solutions to address identified gaps. Results Participants reported a number of limitations with current guidance including a lack of detail on 'how' to adapt, limited information on adaptation of implementation strategies and a number of structural issues with the wording and recommended order of adaptation framework elements. Participants noted that the generation of overarching principles that could be applied together with existing frameworks and/or related resources provides a practical way of supporting adaptation decisions in practice. Finally, participants identified a number of opportunities to further advance the field, including the building of an empirical evidence base on adaptation, advancing adaptation methods and developing data collection systems to capture adaptations. Conclusions This study provides an overview of expert views regarding existing frameworks for adaptation and their ability to be applied in practice. It identified a number of limitations, and suggests overarching principles to be applied when adapting public health initiatives.

\section{Background}

To achieve intended public health benefits from health research, evidence-based interventions and programs (EBIs) need to be adopted, implemented at scale and sustained in real world clinical or community practice. Many public health EBIs, however, are designed to address the needs of a specific target population within a particular context, and as such implementation in diverse settings, or at scale, are likely to necessitate modifications to account for potential population and contextual differences and operational challenges [1]. Such modifications are typically undertaken to address the lack of fit between tested EBI and implementation approaches and provider and target audience characteristics, capabilities, capacity, and resources [2].

The process of modifying an EBI and/or implementation strategies prior to or during the course of implementing an EBI is known as "adaptation" [1]. Adaptation is increasingly recognised as an important process to facilitate adoption and ongoing implementation of an EBI in public health practice $[3,4]$.

Adaptation has been suggested to improve external validity and potentially enhance intervention impact [5] resulting in better public health outcomes. Specifically, there is an increasing evidence-base 
highlighting the benefits of adaptation to the implementation process, with some empirical evidence describing improved acceptability and adoption among providers and recipients following adaptations [6, 7]. While there is recognition of the potential benefits of adaptation, there is some evidence that when poorly planned or unintended, adaptation can inadvertently reduce the impact of any EBI by modifying or reducing the fidelity of core components, such as intervention dose, mechanisms (underlying theory), and content [8].

To maximize the potential benefits of adapting public health programs, numerous frameworks have been developed to provide guidance for both practitioners and researchers on how to systematically approach adaptation of public health programs into new settings or for scale up [9]. A recent (2018) scoping review by Escoffery and colleagues [9] summarized 11 common elements outlined in 13 adaptation frameworks applied in non-clinical settings to provide general guidance regarding the adaptation process. These common elements were: assess community; understand the intervention; select intervention, consult with experts, consult with stakeholders, decide what needs adaptation, adapt the original program, train staff, test the adapted materials, implement, and evaluate [9]. Another recent review by Wang and colleagues (2018) [10] examined frameworks that could be used to adapt guidelines in clinical and public health settings, and provided similar broad recommendations to those outlined by Escoffery et al. These reviews, to our knowledge, provide the most recent and comprehensive synthesis of frameworks in research and practice, and outline common steps for undertaking adaptation of public health interventions. While the purpose of the individual frameworks included in the reviews vary, the synthesis focused on describing practical adaptation steps and as such provides the best available guidance in the literature for those interested in undertaking adaptation.

The ease and usefulness of applying such frameworks to real-world public health adaptation processes however is unknown [9]. Unless such frameworks are utilised by public health policy makers and practitioners, they are unable to yield improvements in public health policy or practice. Previous reviews have identified potential challenges with applying existing frameworks including the lack of detail, limited consideration of how to account for contextual factors as well as poor usability for end-users who operate in environments of time and resource constraints [9] .

\section{Aims}

This study aimed to: i) explore researchers' and practitioners' views on adaptation guidance as summarized in two recent scoping reviews by Escoffrey et al. (2018) and Wang et al, $(2018)[9,10]$, ii) identify potential improvements to increase the practicality of adaptation frameworks in real world settings and iii) identify future opportunities to advance the adaptation literature to achieve better public health outcomes.

\section{Methods}

\section{Design and participants}


A pragmatic qualitative study using elements of participatory research and content analysis was conducted [11]. The study used a modified focus group design, including an elicitation exercise (World Café) and Appreciative Inquiry activities [12]. Such qualitative approaches are recommended for studies where the aim is to examine and understand different perspectives of multiple stakeholders $[11,13]$.

The activities were undertaken on the second day of a two day-long workshop focusing on optimisation and adaptation of public health initiatives. The workshop was conducted in October 2018 and hosted by researchers from the by researchers from the University of Newcastle, Australia as part of a National Health and Medical Research Centre (NHMRC) Centre for Research Excellence in Implementation for Community Chronic Disease Prevention.

Question guides and materials were pilot tested and revised based on feedback from a group of public health research and implementation specialists. Participants were emailed a formal invitation, study information (including the research team's reasons and interests in the research topic) and consent form prior to the workshop. Follow-up emails were sent within the week to participants who did not return a consent form. Consenting participants were required to attend the workshop in Newcastle, Australia. The research team subsidised the costs of international participant attendance.

Participants were purposely recruited to represent three broad groups involved in the adaptation of public health initiatives in Australia and internationally: i) research experts (implementation scientists, behavioural scientists, public health researchers); ii) methodologists(health service research economists and biostatisticians); and iii) policy and practice experts (public health policy makers and health practitioners from government and intermediary organisations). Equal numbers of individuals representing the three groups were approached with some individuals representing multiple groups. Participants included representatives from Cochrane Public Health group, the Campbell Collaborations Knowledge Translation and Implementation Group, The Society for Implementation Research, the European Implementation Collaborative, World Health Organisation Collaborating Centre on Physical Activity, Nutrition and Obesity, the Centre for Evidence and Implementation, the Australian Prevention Partnership Centre, the New South Wales (NSW) Ministry of Health and representative from population health units in NSW, Australia. Representatives from nine academic and/or research institutions from Australia, Canada and the United States were included.

\section{Sample size}

For the conduct of focus groups, ideally four to eight participants are included in each group [11, 13]. Additionally, there should be at least two focus groups of participants with similar backgrounds/qualifications [11, 13]. We approached 33 individuals in order to achieve a sample of 30 (four similarly sized groups of each: research and methodology experts and policy and practice experts).

\section{Ethics approval}


The study was approved by the University of Newcastle's Human Research Ethics Committee (H-20180306). Written consent was received from all participants and confirmed during the day of the workshop.

\section{Data collection}

The workshop sessions took place over six hours. Each activity lasted between one and two hours with breaks in between. The workshop was co-facilitated by research team members with expertise in implementation science (LW, SY) and qualitative and mixed methods (KB).

To address the first aim of exploring researchers' and practitioners' views on adaptation frameworks, the facilitator presented key findings from two recently published adaptation scoping reviews $[9,10]$. The scoping reviews described common elements and a resource for researchers and practitioners to support the adaptation of public health initiatives. Following presentation of the scoping reviews, two experienced health promotion practitioners presented case studies on adaptation of real-world public health initiatives. The case studies were purposefully selected to provide examples of adaptation to an EBI and its implementation strategies in the context of public health practice. Following the presentation, a panel of three experts (including practitioners, researchers and policy makers) discussed their experience of adaptation and potential application of frameworks.

Subsequently, the World Café method was used to familiarise participants with common adaptation framework elements [9] (assess community; understand the intervention; select intervention, consult with experts, consult with stakeholders, decide what needs adaptation, adapt the original program, train staff, test the adapted materials, implement, and evaluate) and to elicit views regarding their application in practice. World Café is composed of elements of: familiarising participants with the topic, engaging in a cyclical group work and collaboratively drafting answers to topics of shared interest. It is a recommended method used to elicit broad insights, foster interaction and dialogue, and to surface the collective wisdom of diverse individuals regarding issues that matter to them [12].

As part of World Cafe, participants formed six small groups, each comprised of both researchers and policy and practice experts. Each small group was assigned to a 'station' consisting of one to two different adaptation elements. Each group was asked to write down practical instructions on how to operationalise the assigned elements in practice. Groups were advised to work creatively, listen for individual contributions, and to keep recommendations pragmatic and suitable for a public health program context. Groups rotated across all 'stations' ('adaptation elements') and contributed to each other's ideas. Upon rotating across all 'stations', the groups returned to their original 'stations' to reflect on everyone's contributions and presented the findings to the larger group.

For the second and third aim of soliciting improvements and identifying future opportunities to advance practice, following the World Café, we facilitated structured focus group discussions and small group activities using Appreciative Inquiry. Appreciative Inquiry is a way of focusing discussions on imagined possibilities rather than problems or issues of the past [12]. In our group process, Appreciative Inquiry was used to elicit participants' suggestions for practical, detailed solutions to recognized gaps [12], and 
their ideas on how to make current adaptation guidance more useful (with or without relying on adaptation frameworks) and move the field forward in terms of undertaking adaptation.

\section{Data capture}

Data were collected via structured notes taken by at least two trained note-takers, facilitators, and those drafted onto flip-charts by participants during small group exercises. Sessions were not audio-recorded. Photos of participants' notes were also taken throughout the workshop.

\section{Data analysis}

Consistent with recommendations, data analysis began during the workshop and continued post workshop $[11,13]$. To help with rapid ongoing data analysis, in addition to the notes taken by two notetakers, one researcher skilled in qualitative methods (KB) took additional notes throughout the workshop and made key observations related to particular activities and discussions. After the workshop, KB consolidated all notes and photos from the workshop and organised them in to the qualitative software package NVivo 12 (QSR, Victoria, Australia). A five step thematic analysis was conducted that included: 1) reading; 2 ) coding; 3 ) displaying; 4) reducing, and; 5) interpreting data [13]. The core research team read the consolidated notes twice, first through the lens of the pre-defined questions/activities, and second to capture key themes that emerged during the activities [11]. Themes were then grouped by broad aims, rather than activity. For example, participants' views on available adaptation frameworks (i.e. limitations etc.), expressed at different points throughout workshop sessions, were grouped together. A coding tree was then developed, and data were coded into broad themes and preliminary categories noted within each theme. Based on this synthesis, within four weeks post-workshop KB prepared a summary report of preliminary findings. Feedback from participants and the larger research team was sought and received via email and in face-to-face meetings. In consultation with the core research team KB then refined themes and interpreted findings. Three consecutive drafts of the refined themes and interpretations of data were presented to workshop participants and the larger research team to seek additional feedback in a manuscript form. Multiple steps were taken to strengthen the rigour of qualitative data analysis and interpretation, including using a team approach to data analysis and interpretation, being sensitive to divergent views and opinions, and having a clear audit trail [14].

\section{Results}

\section{Participant characteristics}

Twenty-four of 33 invited individuals participated in the study. Participants included men (10) and women (14); policy and practice experts $(n=11)$ and research experts $(n=13)$. Workshop attendees held appointments across seven universities or research institutes internationally. Three individuals declined the invitation to participate as they were unable to attend the face-to-face workshop. Six consenting individuals were unable to attend this particular component of the workshop. 


\section{Key findings}

Emerging themes were grouped into three categories according to the study aims: A) experiences with and limitations of adaptation frameworks; B) views on increasing framework utility and practicality in real world settings; and C) opportunities for future research to enhance impact of adaptation to improve impact of public health programs. These are described in detail below, with de-identified quotes from participants illustrating key arguments.

\section{A). Experiences with and limitations of adaptation frameworks}

Participants discussed their experiences in using existing adaptation frameworks and their views on the limitations and applicability of such frameworks in practice.

\section{i. Available frameworks lacking in detail on 'how to' adapt in practice}

Broadly, participants agreed that the framework elements as summarised in the scoping reviews were lacking in detail. Most of the 11 elements found in the frameworks were not considered to be described in ways which help practitioners implement them into practice. For instance, it was not well understood how to 'decide what needs adaptation', or how to 'implement' or 'evaluate', despite some attempts to explicitly provide guidance by the frameworks. While there was a recognition that such details may be implied, a more explicit description of the adaptation elements that were applicable to practitioners was suggested to be required if the frameworks were to guide practice.

A lot of things/steps are assumed, so maybe we need to be more explicit (public health/behavioural scientist)

Although it looks like these are new things, but they may be framed/titled something else and not necessarily missing... Make them explicit. Even if they are not needed [for the particular project], it is still good guidance... (public health/behavioural scientist)

Despite this, participants also suggested that an overly prescriptive framework was unlikely to be useful for practitioners. Some flexibility in how the frameworks are applied was needed, depending on available resource/infrastructure of the 'end-user' or organisation undertaking the adaptation. 
Adaptation occurs mostly at a service level, where they pick and choose what they are implementing based on resources.... an overly technical document is unlikely to be useful (practitioner)

Frameworks [often] provide guidance on what is optimal, not necessarily what's feasible or desirable in practice (public health/behavioural scientist)

.... a trap looking at the very planned instead of what actually happens in the real world, which are the important things (practitioner)

The above quotes illustrate a tension between the recognised need to provide detailed guidance on 'howto in order to make the frameworks more useful, and the reality of often unplanned day-to-day adaptation decisions by practitioners. Participants recognised that while current framework guidance may be too broad, and hence not useful for those undertaking adaptations, producing an 'overly technical document' that requires highly technical skills and increased resources for the operationalisation of adaptation would likely to limit its feasibility to apply in practice. Further, participants noted that guidance needed to be flexible and practical and be considerate of real-world constraints. Consequently, it was noted that future guidance documents on adaptation would need to better consider contextual factors (e.g. level of expertise, role, skills of the individual undertaking adaptation) that impact on users' decision-making and ability to undertake adaptation if such documents were to be useful for practitioners.

Delivery partners will change essential components if resources and capacity force them to (implementation scientists)

I don't see the list as being relevant to a service delivery unit or to final level end user, for example schools... they would not use this. The utility of the framework hinges on [understanding] context (practitioner)

\section{ii. Current adaptation guidance does not adequately cover implementation}

A further theme of discussion related to the lack of explicit guidance on when and how to apply the elements of adaptation to EBI implementation strategies. To ensure EBIs are adequately implemented and embedded into real world practice, effective implementation strategies are needed. Current adaptation frameworks however provide limited guidance on selecting, delivering, measuring and understanding the impact of implementation strategies. Participants noted the 'duality' between the EBI itself (i.e. the public health intervention with established efficacy) and the implementation strategy (i.e. the strategy to support implementation of the EBI into practice), something which is not explicitly acknowledged in current frameworks. Participants recognised them as distinct components of the 
adaptation process, requiring consideration both individually and concurrently. Differences in 'core components' and mechanisms of both an EBI and its implementation strategy were discussed. Core components in the adaptation literature refer to components of the interventions that are necessary to lead to the intended positive outcomes (on an individual, provider, systems level) and in principle should not be adapted [3]. As such, determining core components of the EBI and implementation strategy via mechanism analysis was seen as a critical to informing the adaptation process:

There is always a duality with EBI and implementation... should do mechanisms analysis (separately) and look at different designs for how we can test these. (public health/behavioural scientist)

There is a distinction between essential components of implementation delivery approach and of EBI, different measure of each and different process and outcomes- all of these things need to be measured... For implementation there are different stakeholders, two very distinct development approaches and very distinct evaluation approaches. (public health/behavioural scientist)

The second quote reflects an opinion shared by many, that while there may be some similar elements to guide adaptation of interventions and of implementation strategies, the mechanisms, stakeholders involved, associated outcome measures and evaluation approaches will be different for these two distinct components. It was also noted that for EBIs/policies/guidelines with high fidelity requirements, selection and adaptation of implementation approaches may be particularly important.

\section{iii. Structural limitations including wording and ordering of common elements}

The structure of available adaptation frameworks [9] was also discussed. Participants noted there were some missing elements, such as 'engage with stakeholders' (to precede stakeholder consultation), or 'decide whether to adapt' (to precede a decision on what requires adaptation). Some elements were found to be duplicative and/or overlapping, for example engaging stakeholders and experts was thought of as one element instead of two, and training staff was considered not a separate element, but rather a part of the implementation process. Participants also noted that adaptation in practice was an iterative and cyclical process, rather than the linear model depicted by the current guidance. There was some acknowledgment, however, that for the sake of guidance, some simplification of thinking and presentation of adaptation elements conceptually, as linear, was needed (i.e. a list of ordered adaptation elements as opposed to circuitous process). 
How do we turn it [into] a practical [resource] as we have got an academic model? There is a gap there... we need something practical (to support service decisions)

(implementation scientist)

We want to get to the point where policy makers know what to do... Linear is useful and is simple (implementation scientist)

The need to simplify adaptation frameworks was discussed hand in hand with how to make any framework more accurately represent actual adaptation steps and processes in practice. Some suggested that this might entail working closely with those implementing the intervention to streamline the recommended elements and then to furnish it with real-life examples that are relevant to those who adapt on the ground.

Giving real-world examples about what steps would look like. (We need some) flexibility with which steps to use and how they have been applied, to help show people the relevance of the element and how to apply it (implementation scientist).

Yes, this is a critical point, [the guide] needs to be iterative with examples from different settings, so people can understand it (public health/behavioural scientist)

\section{B) Views on increasing framework utility and practicality in real world settings}

While participants noted that some improvements to include additional detail and refinement of the structure and wording of current recommendations, there was agreement that most of the existing elements [9] were important and relevant. The main suggestions on how to improve the utility of current adaptation frameworks were: i) better consider decisions on how to implement in the adaptation process; and ii) identify guiding principles of adaptation. This was incorporated into a list of overarching principles, which was suggested by participants as important to consider during the adaptation process. These findings are described below.

i.Consider decisions on how to implement in the adaptation process 
Some participants proposed that instead of thinking of adapting intervention and adapting implementation strategies as two separate processes, it could be considered as 'two cycles'.

[adapting intervention and adapting implementation] Shouldn't be considered as stepwise, linear... and not two separate columns, but two integrative cycles (practitioner)

Participants also discussed when to consider implementation approaches as part of adapting EBIs.

Same process, such as elements for EBI and implementation [are used] at the start when comparing what is being done originally. This needs to be done again after adaptation of program... Revisit implementation after adaptation is complete. (public health/behavioural scientist)

The above quote reflects a view shared by some participants, that processes related to adapting the intervention and implementation strategy (where known) should be considered concurrently and then reviewed again when the EBI has been adapted.

ii.Identify guiding principles of adaptation rather than produce an overly prescriptive framework

There was a general consensus that there should be a focus on identifying a set of overarching principles of adaptation, which could then be used together with a more prescriptive framework.

We need to determine key principles first, rather than taking an encyclopaedic approach. (methodologist) We need pragmatic, overarching instructions for system changes (first) and (then can) operationalise them to specific setting/system. (practitioner)

It was proposed that the adaptation process and steps could then be tailored in collaboration with practitioners and end-users involved in the adaptation process, to the specific context and setting within which a program is being adapted. By co-designing with practitioners and tailoring to their needs, and by 
feeding consumer views into the process of adaptation, the guiding adaptation framework is likely to be practical but not overly prescriptive.

Is it practitioners we really need to hear from? If this is the real audience, then in the first instance we should hear back from them (public health/behavioural scientist)

ii. Important overarching 'principles that could be considered in future adaptation processes

Participants recommended six overarching principles as important when considering adaptation in the real world.

1. Identify parameters/drivers of adaptation-perhaps as a first step, or as part of a needs assessment. Parameters relate to boundaries or the context in which adaptation may occur (e.g. who is making adaptation decisions, what skills are available, characteristics of the $\mathrm{EBI} /$ implementation strategies, evidence-base); drivers relate to factors which may determine the need for adaptation (i.e. reasons for adaptation including lack of fit with end-user/community needs, cost restrictions, scale of the intervention). This would allow practitioners to decide whether adaptation is needed, reasons for adapting and what needs to be adapted. The framework developed by Stirman and colleagues [5], which classifies the adaptations into various levels including 'whom' is making a modification, 'what' is being modified (i.e. content, context, or training and evaluation) and at 'what level of delivery' will the modifications be made to was suggested as particularly useful to guide these considerations.

Drivers of adaptation are really important as they guide how to approach (public health/behavioural scientist)

[let's think about] entry strategy... why are we adapting? (practitioner)

Parameters determine level, approach is crucial for adaptation (public health/behavioural scientist)

Consider the work of Stirman... It addresses issues such as by whom, what level, context modification, what has been modified, why. The process of adapting EBI will then require less work and guidance (implementation scientist). 
2. Explicitly identify the implementers (i.e. who will implement the adapted EBI) and implementation strategies early on in the process of adaptation and consult in an ongoing way

Identification of implementer is a critical step as they can communicate to us what will work/ what won't throughout the whole process (practitioner)

3. Identify and engage with relevant stakeholders (e.g. providers, policy makers, practitioners, program implementers) early on, and throughout the adaptation process

(We need to)... engage appropriate stakeholders before consultation. After an appropriate level of engagement, this will help with consultation process. (public health/behavioural scientist)

4. Commence adaptation by selecting an adaptation framework and/or using other available guidance/resources. Tailor application of framework to the end-user context and need and allow for flexibility in applying the framework/adaptation process. Terminate the adaptation process where necessary.

Allow flexibility in applying framework ... need to consider process for getting out/stopping (public health/behavioural scientist)

Put in place termination process .... Like a stopping rule (practitioner)

5. Consider costs of implementing the adapted strategy. This could include an explicit economic assessment/pre-modelling of costs/resources needed.

Need to understand the resources needed to implement the adapted EBI. First, undertake economic assessment...pre-modelling to see if it's cost effective and what budget is needed, and then [understand] 
health service impact... can health service afford to implement the adapted version? Economic modelling guidelines for how to do it [understand costs] are not good or do not exist. (methodologist)

6. Conduct both evaluation and ongoing monitoring of both the adapted EBI and implementation strategy.

Monitoring and sustainability currently missing from frameworks (methodologist)

\section{Opportunities for future research to enhance impact of adaptation on public health programs}

Participants also identified a number of opportunities to undertake research to advance the field of adaptation. Opportunities identified were:

Undertake empirical research to explore/examine the impact of adapting different components of an $\mathrm{EBI} /$ implementation strategy on efficacy and cost outcomes. This provides the opportunity to understand the usefulness of applying formal adaptation frameworks, recognising that many adaptations can be unintended. Further, there is a need to better understand the role of cost/formal economic analysis in supporting the adaptation process.

A lot of [adaptation] research is theoretical - we need to start establishing creative ways of building the empirical evidence. Possible to bed process within existing trials and do comparisons. (Implementation scientist)

[we need to] start understanding the cost and risk in finding a cheaper (adapted) way of delivering a service... for example a nurse instead of a practitioner. To what extent is improved cost (worth) risking the effectiveness? (Implementation scientist)

Cost, budget, financial theme not covered [in current frameworks] (practitioner) 
...need to understand the resources needed, using economic assessment: pre-modelling to see if it's cost effective and what budget is available; and understanding health service impact - can the service afford to implement?(methodologist)

Examine methods that identify core/non-core components of an intervention and its implementation strategy. Introduce formal mechanistic processes where possible (e.g. head to head trials, mechanistic evaluations, mixed methods).

Mechanism analysis is critical for adaptation of implementation strategies... Intensive and tailored strategies make it hard to have one size fits all, which is challenging for scaling. Mixed methods approach needed (public health/behavioural scientist)

Good implementation is evident within efficacious RCTs, so a mechanism analysis should be done [in situations of high implementation fidelity] to identify main contributors for effect of the EBI. This would show what the real core elements are, not the necessarily those hypothesised (practitioner)

Develop systems or data collection processes to capture and measure planned/unplanned adaptations/fidelity to the inte

rvention and implementation strategies. This can provide contextual information on how adaptation may affect the research translation process. The classification framework developed by Stirman et al was again suggested as a useful way to classify any modifications made to an EBI or implementation strategy.

A main challenge for our service is the ability to systematically capture data. (We are) currently running a large implementation trial [with processes] to capture adaptation using Stirman framework to work out where adaptation is occurring at each level (practitioner)

\section{Discussion}

This study initially sought to seek researchers and practitioner's views on how to operationalize common elements described in adaptation frameworks to provide specific guidance on how to undertake adaptation in practice. While participants agreed that available elements were lacking in detail on 'how' to adapt, there was an overall reluctance to suggest a prescriptive text as this was perceived as not useful to end-users. Further, while some structural improvements to current frameworks were proposed, participants noted that most elements included in existing guidance were important and could be included in the adaptation process if there were available skills, expertise and resources. Although this 
was in contrast with views that there was insufficient detail in current frameworks, participants proposed principles that should be considered in practice to inform adaptation decisions and processes that took into account implementation context and cost. The consideration of such principles, together with existing frameworks or other available resources such as IM-Adapt, an online program to support users undertake adaptation [15], is likely to provide a more practical way of supporting those seeking to undertake adaptation of public health programs.

The tension between the need for more detailed guidance and the need to allow flexibility and consider available skills, interest and capacity of those seeking to undertake adaptation (i.e. practitioners, policy makers) was prominently discussed during the workshop. Such discussions could be considered similar to debates in the medical literature regarding 'evidence-based medicine' and the role of individual clinical expertise in decision making and practice $[16,17]$. The medical field has gradually grown to recognize the strengths and limitations of both paradigmsand efforts to break down the divide and incorporate both evidence and professional judgement in practice is now widely accepted [18]. Similarly, in public health and health services, approaches such as 'co-creation', where knowledge generation and application is carried out alongside stakeholders, practitioners and end-users has been proposed as a way of overcoming the gap between practice and research, and has shown potential to generate evidence and guidance to inform on the ground practice [19]. Participants in this study similarly discussed the need to continuously engage practitioners and end-users not only when undertaking adaptation but critically in future iterations of producing guidance for adaptation. Future development, expansion and application of the framework elements need to closely consider who the end-user of the guidance is, and what capacity/infrastructure/training is available to undertake suggested processes.

Participants also identified multiple opportunities to further advance the field of adaptation research including generating empirical evidence, advancing adaptation methods and developing data collection systems to capture unplanned/unintended adaptations. Some of the recommendations would require a high level of technical skills and expertise, and for these reasons are unlikely components of practical adaptation guides. However, they highlight potential areas for researchers to work closely together with those undertaking adaptation to embed measurement and evaluation approaches in practice. In a call for determining core/non-core intervention components, participants highlighted that a better understanding of the processes and methods surrounding mechanistic evaluation of EBIs and implementation strategies are needed. While some guidance is available [20], working closely with end-users in planning and developing causal models and in embedding high-quality, non-invasive data collection methods in intervention evaluation is needed to answer these questions. Additionally, where routine sources of practice or patient data are available, planning and embedding head to head or factorial trials provides a way of further elucidating core/non-core intervention components - particularly for multi-strategy interventions, similar to that demonstrated in audit and feedback laboratories [21].

\section{Strengths and limitations}


This study sought to seek input from a broad range of participants representing organisations that were interested in undertaking or advancing the science of adaptation. A high level of participation from all individuals in the group was achieved on the day of discussion and in shaping subsequent results. The qualitative approaches applied in this study (World Café and Appreciative Inquiry) provided us with a flexible method to elicit participant views, building on previous responses and responding to participants' input. The World Café approach [12] lent itself well to exploring groups' experiences around adapting public health interventions in practice and mapping out relationships between concepts. In addition, participants' ideas on how to make current adaptation guidance more useful and how to move the field forward in terms of undertaking adaptation was effectively elicited with Appreciative Inquiry [12]. While we moved away from the original intent of the discussion, we suggest that the qualitative, exploratory methods used in this study significantly contributed to the richness of study outcomes and their usefulness for practice. Collectively, this provides us with a range of perspectives reflecting both research and practice views.

This study had a number of limitations. We did not audio record the discussion on the day and relied on note takers given that format of the day included many breakouts into small groups. As such quotes included in the study are not completely verbatim but have been approved by participants. While we sought to discuss adaptation broadly, certain type of adaptations such as cultural adaptations may have necessitated further inquiry and specific discussion. Cultural adaptations were noted by participants as well and within the literature [22] as having different considerations and principles that may not have been captured in this discussion.

\section{Conclusion}

The study summarizes participant's views of existing guidance for adaptation of public health initiatives. While participants identified a number of limitations with available adaptation guidance including a lack of detail, producing an overly prescriptive text was not seen as useful for end-users. Instead, we propose a broad set of overarching principles including identify parameters/drivers of adaptation, explicitly identify implementers, identify and engage with relevant stakeholders, commence adaptation, consider costs of implementing the adapted strategy, and conduct both evaluation and ongoing monitoring. The set of overarching principles outlines practical considerations for adaptation that could be used with existing frameworks and/or resources to support adaptation in real-world contexts.

\section{Declarations}

\section{Ethics approval and consent to participate}

All procedures performed in studies involving human participants were in accordance with the ethical standards of the institutional and/or national research committee and with the 1964 Helsinki declaration and its later amendments or comparable ethical standards. Informed consent was obtained from all individual participants included in the study. 
The study received low risk ethical approval from the University of Newcastle's Human Research Ethics Committee, Protocol No H-2018-0306.

\section{Consent for publication}

Consent for publication of de-identified quotes was obtained from all study participants.

\section{Availability of data and material}

The datasets used and/or analysed during the current study are available from the corresponding author on reasonable request.

\section{Competing interests}

The authors declare that they have no conflicts of interest.

\section{Funding Sources}

Workshop was supported by funding provided by the NHMRC Translating Research Into Practice Fellowship scheme, institutional funds (The University of Newcastle) awarded to LW and funds from Australian Research Council Discovery Early Career Award awarded to SY. Infrastructure support was provided by the Hunter Medical Research Institute.

SY is supported by an Australian Research Council Discovery Early Career Award (DE170100382). MK is supported by a NHMRC TRIP Fellowship (APP1150476).

NN is supported by a NHMRC TRIP Fellowship (APP1132450) and a Hunter New England Clinical Research Fellowship; LW is supported by a NHMRC Career Development Fellowship (APP1128348), Heart Foundation Future Leader Fellowship (101175) and a Hunter New England Clinical Research Fellowship; RS is supported by a NHMRC TRIP Fellowship (APP1150661).

The research team subsidised the costs of international participant attendance.

\section{Authors' contributions}

SY (PhD) put forward the idea for the study and the conceptual underpinnings of the paper. During the workshop SY co-facilitated various group discussions and participated in the analysis of qualitative data. SY led the drafting of the manuscript and in particular contributed to introduction, discussion and conclusions sections.

$\mathrm{KB}(\mathrm{PhD})$ proposed the qualitative methods employed by the study, facilitated various discussions during the workshop, trained note takers, led the data analysis process, prepared drafts of key findings, sought and incorporated participants' feedback. KB drafted methods and results sections of the paper. 
All other participants participated in the workshop and provided written input through elicitation exercises. All provided feedback post-workshop on the preliminary themes from the study and contributed to multiple iterations of the manuscript. In addition to the above, the internal research team (LW, KB, AG, SMc, MK, NN, RW, RH, RS and SY) assisted in preparing and running of the workshop. In addition to the above, $\mathrm{LW}(\mathrm{PhD})$ contributed to the conceptual underpinning of the study.

All authors contributed to and approved the final version of this manuscript.

\section{Acknowledgements}

The authors acknowledge those workshop participants who did not contribute to the write-up of this paper: Professor Heather McKay, Dr Jeremy Grimshaw, Dr Cara Lewis, Dr Jed Duff, Ms Penny Reaves, Dr Robyn Mildon, Dr Chris Doran and Miss Samantha Gray.

We also acknowledge Ms Courtney Barnes, Ms Alison Brown, Dr Meghan Finch and Dr Alice Grady who helped organise the workshop and were key note takers during the workshop, and Ms Melinda Phillips for her assistance with logistics of the workshop.

\section{References}

1. Escoffery, C., et al., A systematic review of adaptations of evidence-based public health interventions globally. Implementation Science, 2018. 13(1): p. 125.

2. Moore, J.E., B.K. Bumbarger, and B.R. Cooper, Examining adaptations of evidence-based programs in natural contexts. Journal of Primary Prevention, 2013. 34(3): p. 147-161.

3. Chambers, D.A. and W.E. Norton, The Adaptome: Advancing the Science of Intervention Adaptation. American Journal of Preventive Medicine, 2016. 51(4): p. S124-S131.

4. Graham, I.D., et al., Moving knowledge into action for more effective practice, programmes and policy: protocol for a research programme on integrated knowledge translation. Implementation science : IS, 2018. 13(1): p. 22-22.

5. Stirman, S., et al., Development of a framework and coding system for modifications and adaptations of evidence-based interventions. Implement Sci, 2013. 8(1): p. 65.

6. Sundell, K., et al., Novel Programs, International Adoptions, or Contextual Adaptations? MetaAnalytical Results From German and Swedish Intervention Research. Journal of Clinical Child \& Adolescent Psychology, 2016. 45(6): p. 784-796.

7. Whittemore, R., A systematic review of the translational research on teh Diabetes Prevention Program. Translational Behavioral Medicine, 2011. 1(3): p. 480-91.

8. McCrabb, S., et al., Scaling-up evidence-based obesity interventions: A systematic review assessing intervention adaptations and effectiveness and quantifying the scale-up penalty. Obesity Reviews, 2019. 
9. Escoffery, C., et al., A scoping study of frameworks for adapting public health evidence-based interventions. Translational behavioral medicine, 2018.

10. Wang, Z., S. Norris, and L. Bero, The advantages and limitations of guideline adaptation frameworks. Implementation Science, 2018. 13(72).

11. Green, J. and N. Thorogood, Qualitative Methods for Health Research, 4th Ed, ed. J. Green and N. Thorogood. 2018: Sage.

12. http://www.artofhosting.org/what-is-aoh/methods/. The Art of Hosting and Harvesting Conversations that Matter. [cited 201815 Sept 2018].

13. Tolley, E., et al., Qualitative Methods in Public Health: A Field Guide for Applied Research, 2nd Edition, ed. E. Tolley, et al. 2016, San Francisco, CA: Wiley: Jossey-Bass.

14. Lincoln, Y.S. and E.G. Guba, Naturalistic inquiry. 1985: Beverly Hills, Calif. London : Sage Publications.

15. The University of Texas Health Science Center at Houston. [cited 2018 1/11/2018]; Available from: https://www.imadapt.org/\#/.

16. Szajewska, H., Evidence-Based Medicine and Clinical Research: Both Are Needed, Neither Is Perfect. Vol. 72. 2018. 8-18.

17. Accad, M., Does evidence based medicine adversely affect clinical judgment? BMJ, 2018. 362:k2799.

18. Karthikeyan, G. and P. Pais, Clinical judgement \& evidence-based medicine: time for reconciliation. The Indian journal of medical research, 2010. 132(5): p. 623-626.

19. Greenhalgh, T., et al., Achieving Research Impact Through Co-creation in Community-Based Health Services: Literature Review and Case Study. The Milbank Quarterly, 2016. 94(2): p. 392-429.

20. Lewis, C.C., et al., From Classification to Causality: Advancing Understanding of Mechanisms of Change in Implementation Science. Frontiers in public health, 2018. 6: p. 136-136.

21. Grimshaw, J.M., et al., Reinvigorating stagnant science: implementation laboratories and a metalaboratory to efficiently advance the science of audit and feedback. BMJ Quality \&amp;amp; Safety, 2019: p. bmjqs-2018-008355.

22. Barrera, M. and F. Gonzalez Casto, A Heuristic Framework for the Cultural Adaptation of Interventions. Clinical Psychology Science and Practice, 2006. 13(4): p. 311-16.

\section{Supplementary Files}

This is a list of supplementary files associated with this preprint. Click to download.

- Additionalfile1COREQ.pdf 